\title{
Enfermedad de Behcet: Estudio clínico y tratamiento en el Hospital Arzobispo Loayza.*
}

\author{
ALZAMORA BARRIOS Blanca E.**; MARTÍNEZ YACTAYO Franklin Y.***
}

\section{SUMMARY:}

Objetives: To determine the frecuency of presentation, as well as, clinical findings, treatment and evolution of Behcet's disease at the Hospital Arzobispo loayza, between 1992 and 2001. Material and methods: This is a retrospective and descriptive study was performed between 1992 and 2001 at the Hospital Arzobispo Loayza. We include outpatients of Ophtalmology and Internal medicine divisions, whan were hospitalized and they had Behcet's disease diagnosis. Results: We report 9 patients with Behcet's disease between 1992 and 2001, 6 females and 3 males, among the 19 - 55 years of age. They presented as clinical findings: recurrent oral aphthous ulcers, genital ulcers, uveitis and others eye lesions, skin lesions, joint pain, gastrointestinal tract involvement and others. Conclusion: The prevalence of this disease in our poblation is low, similar to the rest of the world. Behcet's disease diagnosis is dificult and based in clinical, because it frecuency is low. ( Rev Med Hered 2001; 12: 58-64 ).

KEY WORD: Behcet's disease, oral ulcers, genital ulcers, uveitis.

\section{RESUMEN:}

Objetivo: Determinar la frecuencia de presentación, así como, las manifestaciones clínicas, tratamiento y evolución de la Enfermedad de Behcet en el Hospital Arzobispo Loayza (HAL) durante el periodo 1992 - 2001. Material y Métodos: Se realizó un estudio retrospectivo descriptivo en el HAL durante el periodo de 1992 a 2001. Se revisaron las historias clínicas de los pacientes que estuvieron hospitalizados y que acudieron a consultorios externos de los servicios de Oftalmología y Medicina y, tuvieron como diagnóstico final Enfermedad de Behcet. Resultados: Se presentaron 9 pacientes con Enfermedad de Behcet durante el periodo 1992 - 2001, 6 mujeres y 3 hombres, con edades entre los 19 y 55 años; los cuales presentaron como manifestaciones clínicas: úlceras orales, úlceras en genitales, uveítis y otras lesiones oculares, lesiones en piel, dolor articular, compromiso gastrointestinal, etc. Conclusiones: Se encuentra que la prevalencia de esta enfermedad, en nuestra población es baja, al igual que en el resto del mundo y; se confirma además que, el diagnóstico de esta enfermedad, a pesar de ser clínico, es difícil, porque es una entidad poco reconocida. ( Rev Med Hered 2001; 12:58-64).

PALABRAS CLAVE: Enfermedad de Behcet, úlceras orales, úlceras genitales, uveítis.

\footnotetext{
* $\quad$ Estudio realizado en Servicio de Oftalmología, Dpto. de Cirugía. Hospital Arzobispo Loayza

** Medico asistente del Servicio de Oftalmología, Dpto. de Cirugía del Hospital Arzobispo Loayza. Profesora Asociada del Departamento de Cirugía. Facultad de Medicina Alberto Hurtado. Universidad Peruana Cayetano Heredia.

*** Alumno del $5^{\circ}$ año de la Facultad de Medicina Alberto Hurtado. Universidad Peruana Cayetano Heredia.
} 


\section{INTRODUCCIÓN}

La enfermedad de Behcet es rara, de evolución crónica y con capacidad para producir inflamación a nivel de los vasos sanguíneos del organismo, por lo que se considera una vasculitis sistémica $(1,2,3)$. Causa úlceras orales dolorosas, úlceras genitales recurrentes e inflamación ocular. También puede causar varios tipos de lesiones en la piel, artritis, inflamación intestinal con diarrea e inflamación a nivel de las estructuras del sistema nervioso $(1,4,5)$, tanto central (cerebro, cerebelo, tronco encefálico, médula espinal, meninges) como de los nervios periféricos. Estas lesiones las produce inflamando los vasos sanguíneos, tanto arterias como venas $(1,2)$.

La enfermedad de Behcet comienza generalmente en la tercera o cuarta década de la vida. Sin embargo hay casos descritos de muy diversas edades, desde recién nacidos a octogenarios.

Aunque inicialmente se consideró una enfermedad propia de los países mediterráneos y Japón, actualmente se sabe que la enfermedad está esparcida por todo el mundo, aunque estas áreas geográficas son las que tienen un mayor número de casos. La creciente descripción de casos en los países asiáticos hace que muchos autores relacionen su distribución con las rutas comerciales remotas y le den el nombre de enfermedad de la Ruta de la Seda. La prevalencia de la enfermedad en Estados Unidos es de 0.12 - 0.33 casos por 100,000 habitantes, en Gran Bretaña es de 0.64 casos por 100,000 habitantes, España puede acercarse a los 5 casos por 100,000 habitantes, mientras que en Japón, Corea, China, Irán y Arabia Saudita es de 13.5 - 20 casos por 100,000 y, en Turquía de 80 - 370 casos por 100,000 habitantes. $(2,4,6,7,8,9,10,11,12)$.

La causa de dicha enfermedad no es conocida, pero ha sido relacionada con ciertos microorganismos como estreptococos de diferentes clases, pues antígenos de dichas bacterias han sido localizados en muestras de tejidos de pacientes con la afección y puede ser exacerbada con pruebas cutáneas con dichos antígenos $(1,2,12)$.

El virus herpes simple tipo 1 también parece estar implicado en la génesis de la enfermedad pues su genoma ha sido identificado en leucocitos de sangre periférica de los pacientes $(1,12,13)$.

Se piensa que la enfermedad aparece en personas genéticamente predispuestas que, se ven expuestas a algún agente externo medioambiental. Los pacientes con la enfermedad suelen tener defectos en el sistema inmunológico $(1,2,10,13,14)$. La evolución de la enfermedad de Behcet suele ser intermitente, con períodos de remisión y exacerbación a lo largo de los años, con una tendencia hacia la remisión. Los síntomas pueden durar desde días a semanas, o pueden comportarse de forma crónica durante meses o años. Tienden a causar un gran malestar y pueden provocar niveles de incapacidad importante que interfieren con la calidad de vida $(2,10,15)$.

El paciente es generalmente tratado de cada una de las manifestaciones que va presentando de una manera separada y no es enfocado como un conjunto de síntomas y signos, como un todo, en la mayoría de los casos.

La baja prevalencia hace que esta enfermedad sea poco reconocida y diagnosticada. Además, debemos recordar que el es clínico $(4,6,15)$.

El objetivo de este estudio fue determinar la frecuencia de presentación, manifestaciones clínicas, tratamiento y evolución de la Enfermedad de Behcet en el Hospital Nacional Arzobispo Loayza.

\section{MATERIAL Y METODOS}

Se realizó un estudio retrospectivo descriptivo de los pacientes portadores de la Enfermedad de Behcet. Para esto se revisaron las historias clínicas de los pacientes que fueron hospitalizados y que acudieron a consultorios externos de los servicios de Oftalmología y Medicina del Hospital Arzobispo Loayza (HAL) y tuvieron como diagnóstico final enfermedad de Behcet, durante los periodos de 1992 al 2001.

Fueron seleccionados todos los pacientes que presentaron como diagnóstico final Enfermedad de Behcet y aquellos pacientes que presentaban signos y síntomas según los Criterios Internacionales de la Asociación Americana para la Enfermedad de Behcet $(18,19)$, dados a continuación:

La manifestación primaria:

- Ulceras orales recurrentes en casi el $100 \%$ de los pacientes ( úlceras aftosas dolorosas, con pruebas negativas para Herpes).

Además se deben considerar 2 de las siguientes 4 manifestaciones:

- Ulceras genitales recurrentes (semejantes a las úlceras orales, usualmente dolorosas, con pruebas negativas para Herpes) 
- Manifestaciones oculares o perioculares (Células y fenómeno de Tyndall en cámara anterior, hipopion, uveítis anterior o posterior, trombovasculitis, atrofia del nervio óptico, neurorretinitis, edema de mácula, hemorragias u opacidades en humor vítreo, disminución de la agudeza visual, glaucoma secundario, etc.)

- Lesiones en piel ( Eritema nodoso, pseudofoliculitis, lesiones pápulopustulosas o nódulos acneiformes relacionados con la enfermedad de Behcet, estos últimos, en pacientes que pasaron la adolescencia y que no recibieron corticoesteroides.)

- Positividad del test de hiperreactividad cutánea (Patergia).

La prueba consiste en pinchar con una aguja estéril el antebrazo del paciente. El resultado es positivo cuando la punción causa en 24 a 48 horas, un nódulo eritematoso aséptico o pústula que tiene más de $2 \mathrm{~mm}$ de diámetro. En el sitio de la reacción hay inicialmente una acumulación de neutrófilos, seguida de una acumulación de células mononucleares. La prueba puede ser positiva también en el Síndrome de Sweet (Cefalea, artralgia, leucocitosis y nódulos edematosos dolorosos en cara, cuello y miembros superiores.) y en la piodermitis gangrenosa.

Otras manifestaciones que pueden ser utilizadas en el diagnóstico, pero que no son consideradas parte de los criterios internacionales para la enfermedad de Behcet son:

- Complicaciones en venas: (Tromboflebitis subcutánea, Trombosis venosa profunda, bloqueo de la Vena cava superior y/o inferior, Síndrome de Budd-Chiari )

- Epididimitis ( Hinchazón y dolor a nivel de uno de los 2 testículos).

- Oclusión arterial y/o aneurisma (Ruptura)

- Complicaciones en el Sistema nervioso central (SNC) ( Dificultades en el movimiento o el habla, Pérdida de Memoria, Síntomas y Signos Piramidales, Extrapiramidales y/o Cerebelosos, Pseudotumor cerebral, puede imitar el curso de una Esclerosis Múltiple, Meningoencefalitis, Hipertensión Endocraneana Benigna.)

- Dolor articular (Artralgia o una artritis no destructiva)

- Cefalea severa con rigidez en cuello (probable meningitis aséptica)

- Complicaciones en el Tracto Gastrointestinal (GI) ( Hinchazón, Calambres, Náuseas, Vómitos, Diarreas, Hematemesis, Melena, Lesiones que pueden aparecer en cualquier parte del tracto digestivo.

- En Riñón ( Anormalidades en la función renal, Síndrome Nefrótico, Amiloidosis)

- Inflamación pulmonar vascular y Pleuritis.

- Historia familiar.

Además de las manifestaciones descritas se pueden presentar:

Fatiga, problemas auditivos (Tinnitus, Perdida fluctuante de la Audición, etc.) $(7,8)$. Es posible encontrar arritmias cardiacas asociadas a la enfermedad de Behcet. (9)

\section{RESULTADOS}

Durante el periodo de 1992 al 2001 fueron hospitalizados y acudieron a consultorios externos de Oftalmología y Medicina del HAL, 9 pacientes que presentaban el criterio principal y 2 ó más de los criterios secundarios dados por la Asociación Americana para la Enfermedad de Behcet.

Sieta pacientes quedaron con el diagnóstico final de Enfermedad de Behcet al no encontrarse alteraciones en los diversos exámenes de laboratorio realizados. A 2 pacientes se les encontró una o más etiologías a sus manifestaciones clínicas (Tricomoniasis, Toxocariasis) pero, durante la evolución aparecieron lesiones en órganos y sistemas que, los incluían en los criterios para la Enfermedad de Behcet.

En la tabla $\mathrm{N}^{\circ} 1$, se muestra el año de presentación de los primeros síntomas y la procedencia de los pacientes. En la tabla $\mathrm{N}^{\circ} 2$, se puede apreciar la edad, el sexo y el resultado de las biopsias de piel de los pacientes incluidos. Las manifestaciones clínicas de los pacientes en el curso

\begin{tabular}{|ccc|}
$\begin{array}{c}\text { Tabla No1. Procedencia y fecha de inicio de enfermedad de } \\
\text { los pacientes estudiados. }\end{array}$ \\
\hline PACIENTE & $\begin{array}{c}\text { INICIO DE } \\
\text { ENFERMEDAD }\end{array}$ & PROCEDENCIA \\
\hline A & Mar'92 & Lima \\
B & Abr' '93 & Lima \\
C & Jun' 93 & Callao \\
D & Feb '94 & Lima \\
E & Ene '96 & Huancayo \\
F & Jul'96 & Lima \\
G & Sep '97 & Chiclayo \\
H & Abr' '98 & Trujillo \\
I & Ene '01 & Lima
\end{tabular}




\begin{tabular}{cccl}
\hline $\begin{array}{c}\text { Tabla N2. Edad, sexo y resultado de la biopsia de } \\
\text { los pacientes estudiados. }\end{array}$ \\
\hline PACIENTE & EDAD & SEXO & $\begin{array}{l}\text { RESULTADO } \\
\text { DE BIOPSIA }\end{array}$ \\
\hline A & 32 & M & No realizada \\
B & 26 & F & Vasculitis \\
C & 36 & F & Vasculitis \\
D & 44 & F & Vasculitis \\
E & 55 & F & Vesculitis leucocitoclástica \\
F & 23 & F & No realizada \\
G & 35 & M & Vasculitis \\
H & 19 & F & Vasculitis \\
I & 43 & M & Vasculitis \\
\hline
\end{tabular}

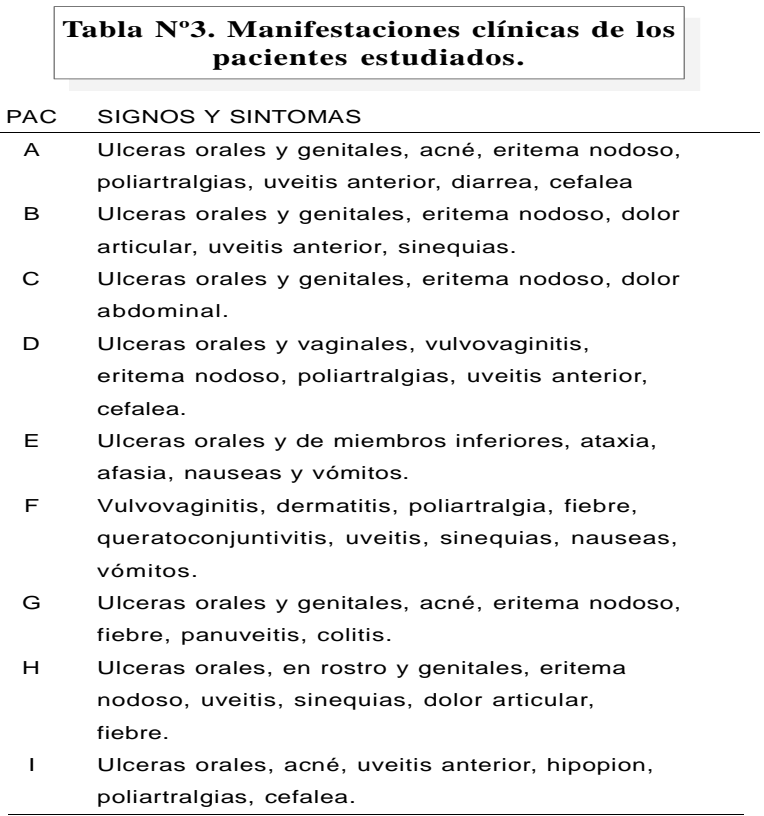

Tabla No4. Tratamiento.

\begin{tabular}{|c|c|}
\hline PAC & TRATAMIENTO \\
\hline A & $\begin{array}{l}\text { AINE, Corticoides tópicos, ciclopléjicos, } \\
\text { prednisona, clorambucil. }\end{array}$ \\
\hline B & $\begin{array}{l}\text { AINE, Corticoides tópicos, atropina oftálmica, } \\
\text { prednisona. }\end{array}$ \\
\hline $\mathrm{C}$ & $\begin{array}{l}\text { AINE, ciprofloxacina, dicloxacilina, eritromicina, } \\
\text { isoniacida, prednisona. }\end{array}$ \\
\hline $\mathrm{D}$ & $\begin{array}{l}\text { AINE, Corticoides tópicos, atropina oftálmica, } \\
\text { prednisona. }\end{array}$ \\
\hline $\mathrm{E}$ & $\begin{array}{l}\text { Ciprofloxacina, metronidazol, metilprednisolona, } \\
\text { prednisona, ciclofosfamida. }\end{array}$ \\
\hline $\mathrm{F}$ & $\begin{array}{l}\text { Ciprofloxacina, acetazolamida, prednisona, } \\
\text { azatioprina. }\end{array}$ \\
\hline G & $\begin{array}{l}\text { AINE, albendazol, atropina oftálmica, prednisona, } \\
\text { ciclosporina, clorambucil. }\end{array}$ \\
\hline $\mathrm{H}$ & $\begin{array}{l}\text { Paracetamol, ciprofloxacina, AINE, atropina } \\
\text { oftálmica, pentofilina, prednisona. }\end{array}$ \\
\hline 1 & AINE, atropina oftálmica, prednisona, clorambucil. \\
\hline
\end{tabular}

AINE: Antiinflamatorios no esteroideos. de la evolución de su enfermedad, se pueden observar en la tabla $\mathrm{N}^{\circ} 3$ y figura $\mathrm{N}^{\circ} 1$.

En la Tabla $N^{\circ} 4$, se muestra el tratamiento que recibieron los pacientes.

\section{DISCUSIÓN}

En este estudio se encontró que 9 pacientes que estuvieron hospitalizados y/o fueron atendidos en consultorios externos de Oftalmología y Medicina del HAL, tuvieron como diagnóstico final la Enfermedad de Behcet.

A pesar de tener un número pequeño de casos, algunos datos concuerdan con lo registrado por otros países que tienen una mayor casuística que la presentada $(13,22,23,33,34,35)$.

La procedencia del paciente es variada, aunque se tiene en consideración que pueden existir factores ambientales comunes que predispongan a la enfermedad; en nuestra casuística, la mayoría proviene de una zona urbana como es Lima. La edad de presentación tiene un rango muy amplio pero, hay una tendencia a concentrarse en la tercera a cuarta década; en el presente estudio encontramos también esta predisposición a concentrarse en la segunda a tercera década de la vida. Hay mayor tendencia a presentarse en pacientes de sexo femenino; nosotros encontramos en el estudio una relación de mujer a hombre que es de 2 a 1 y el resultado de las Biopsias realizadas, mostraba en su mayoría infiltrado inflamatorio agudo y crónico en los vasos sanguíneos de la dermis; concluyendo como diagnóstico vasculitis.

En el presente estudio, no se han encontrado casos de Enfermedad de Behcet en menores de edad (0 - 17 años); sin embargo algunos estudios $(10,14)$ muestran que la Enfermedad de Behcet en recién nacidos se caracteriza por presentar Aftas orales y lesiones en piel que desaparecen espontáneamente antes de los 6 meses. Difiere de los adultos en que es menos frecuente la presentación ocular (18) y aparecen mas bien manifestaciones poco comunes como: Neutropenia, Esplenomegalia, Síndrome de Budd-Chiari (Trastorno circulatorio hepático con obstrucción venosa que produce agrandamiento del hígado, ascitis, circulación colateral e hipertensión portal), infiltrado pulmonar y ruptura de aneurisma de la arteria pulmonar. La transmisión de la enfermedad de la madre al hijo puede ser causada por anticuerpos que ingresan al feto a través de la placenta. Es raramente diagnosticado en niños, pero debe ser considerado como diagnóstico diferencial en los desórdenes inflamatorios con compromiso 
multisistémico.

En el presente estudio, encontramos un compromiso de órganos y sistemas que guarda relación con lo mencionado por otros estudios $(11,17,19,22,32,34,35)$. Encontramos en el estudio: $89 \%$ de úlceras orales, consistente en: aftas y lesiones vesiculares en labios, lengua, paladar duro, úvula y faringe posterior. Los diversos estudios señalan una frecuencia de 50 a $70 \%$, llegando al $98 \%$ durante el curso de la enfermedad.

Nosotros encontramos $77.7 \%$ de ulceras genitales que comprometen en la mujer: vulva y vagina y en el hombre en pene y escroto, similar a otros estudios que señalan una frecuencia de $72-80 \%$ de los pacientes.

El compromiso ocular se encontró en el $77.7 \%$, entre los cuales figuraban uveitis anterior y/o posterior, queratoconjuntivitis, neurorretinitis, hifema, hipopion, sinequias y disminución de la agudeza visual, hallazgo muy similar al encontrado en otros estudios, los que describen una frecuencia de presentación de 67-75\%, haciendo referencia además de complicaciones que pueden presentarse durante la evolución como: cataratas, glaucoma, atrofia del globo ocular y ceguera.

Todos los pacientes incluidos en el estudio presentaron lesiones dérmicas, entre los cuales se consideró el eritema nodoso, acné, lesiones máculo-papulares, úlceras en miembros superiores y/o inferiores y dermatitis. El $66.7 \%$ presentaron compromiso articular, caracterizado por artritis y artralgias mayormente en rodillas y codos, hallazgos muy similares a otros estudios.

El compromiso gastrointestinal se encontró en el $55.6 \%$ de los pacientes. Los mas frecuentes fueron: nauseas, vómitos, dolor abdominal y diarrea. Otros estudios, señalan una frecuencia entre 16 y $25 \%$.

El compromiso del sistema nervioso, caracterizado por ataxia, afasia de comprensión, hipo e hiperreflexia, signo de Babinsky bilateral (+) y cansancio, se presentó en $11 \%$ de los pacientes, similar a otros estudios los que describen una frecuencia de 8 a $20 \%$ y se menciona que aparecen de 1 a 11 años después de las primeras manifestaciones clínicas.

Como la causa de la enfermedad es desconocida, el tratamiento se hace de acuerdo a los síntomas individuales y al momento de su aparición. Los medicamentos están dirigidos a reducir la inflamación o bien a intentar regular el sistema inmunológico $(3,4,17)$.

El tratamiento que recibieron los pacientes fue inicialmente con sintomáticos (en su mayoría antiinflamatorios no esteroideos). En 9 pacientes se utilizó corticoides (Prednisona $1 \mathrm{mg} / \mathrm{Kg} /$ día) e inmunosupresores se utilizaron en 5 pacientes, por tener una respuesta pobre a los corticoides; clorambucil fue el más utilizado, a una dosis de $2 \mathrm{mg} / \mathrm{Kg}$ /día. Azatioprina a dosis de $2-3 \mathrm{mg} / \mathrm{kg} /$ día por vía oral y Ciclosporina a dosis de 3 - $5 \mathrm{mg} / \mathrm{kg} /$ día por vía oral, fueron los otros inmunosupresores utilizados.

El tratamiento utilizado en los diversos estudios $(3,4,12,13,16,17,20,21)$, incluyen corticoides tópicos, antiinflamatorios no esteroideos y analgésicos, colchicina para prevenir brotes articulares o cutáneos de la enfermedad, corticoides sistémicos solos o combinado con clorambucil (30), inmunosupresores (azatioprina, metotrexate, ciclosporina (31), tacrolimus, ciclofosfamida, clorambucil e interferon alfa $2 b$ ), pentoxifilina y dapsona.

El Tacrolimus (FK506): ( Dosis $0.10-0.15 \mathrm{mg} / \mathrm{Kg} /$ día) tuvo un exitoso resultado como un agente Inmunosupresor, antibiótico Macrólido, utilizado para el tratamiento de la Uveítis posterior y la vasculitis retinal en los pacientes que no respondieron a otro inmunosupresor como: la Ciclosporina, por falla en los efectos terapéuticos o por efectos adversos como: Hipertensión, hipercalemia, hiperglicemia, parestesias en dedos, dolor abdominal y diarrea (16).

La Talidomida: parece ser una droga eficaz en el tratamiento de la enfermedad de Behcet tanto a la dosis de $100-300 \mathrm{mg} /$ día. Se reportó un caso en el que se encontró una severa colitis y respondió favorablemente solo a la Talidomida (12). Aunque la mayoría de los estudios sobre esta droga muestran únicamente mejoría de las ulceras orales y genitales. Sin embargo su uso debe reservarse a los verdaderos casos de esta entidad, debido a su gran efecto teratogénico, potenciador de los efectos sedantes del alcohol, barbitúricos y clorpromacina; puede producir además: anorexia, hipotensión ortostática, constipación, fiebre, boca seca, etc.

La enfermedad de Behcet a sido asociada a los antígenos HLA B5 (B51 y B52) y HLA DR5 $(1,2,3,22,23,24,25,26,27)$. En el presente estudio, se realizó HLA B5 a 3 pacientes, siendo el resultado positivo en todos ellos. 


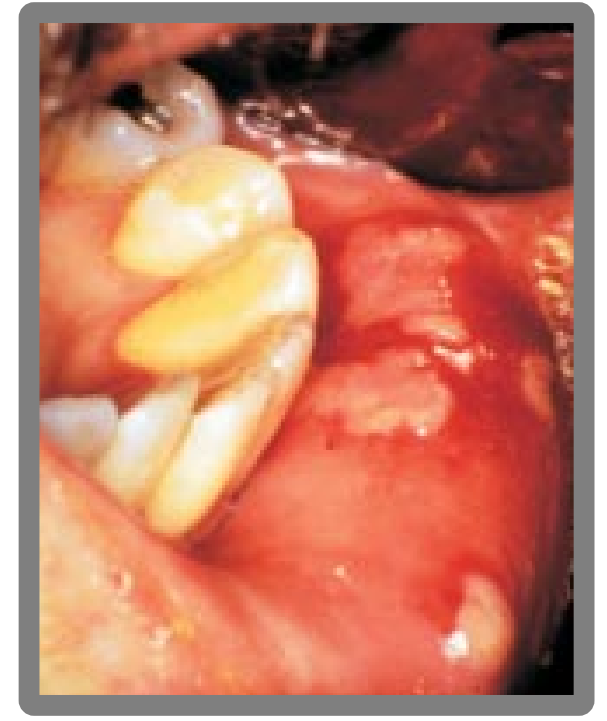

ULCERAS ORALES: Aftas orales

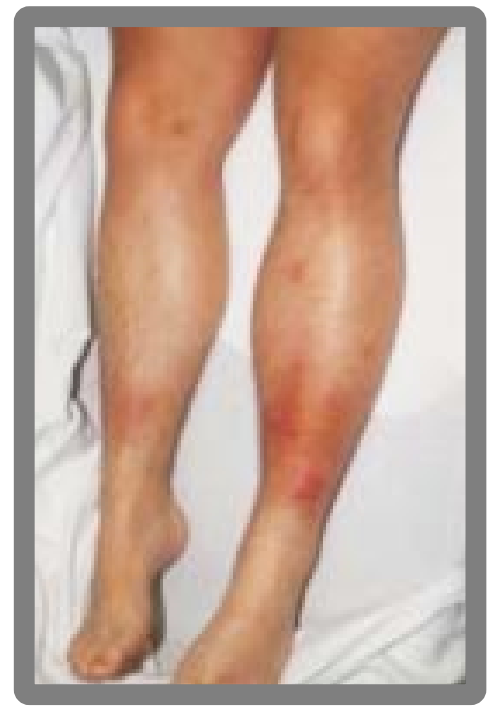

LESIONES EN PIEL: Eritema nodoso

Queda abierta la posibilidad de realizar un Estudio Multicéntrico para determinar la prevalencia de esta enfermedad en nuestra población.

\section{Correspondencia:}

Blanca E. Alzamora Barrios

Av. Alberto del Campo 468 Dpto. 202. San Isidro e-mail: beab@terra.com e-mail: frankmart01@ @otmail.com

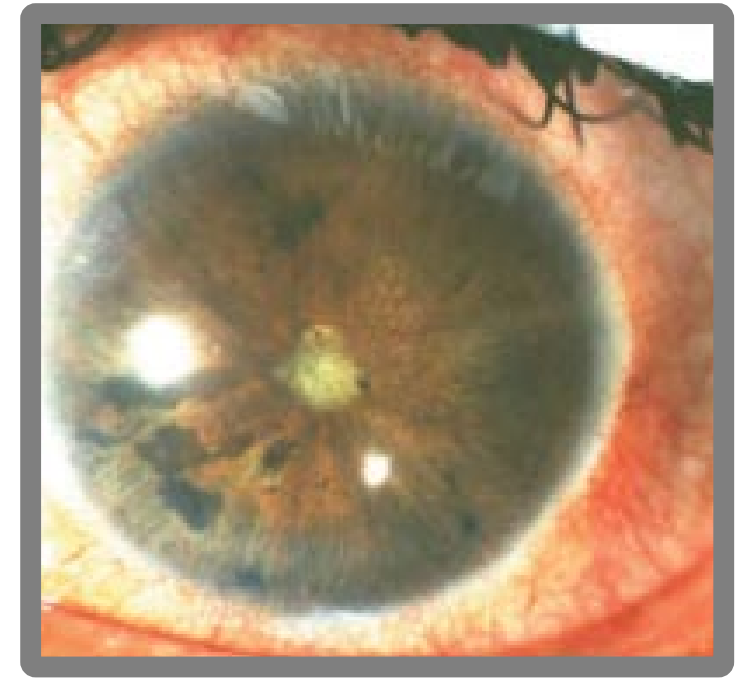

COMPROMISOS OCULAR: Uveítis anterior

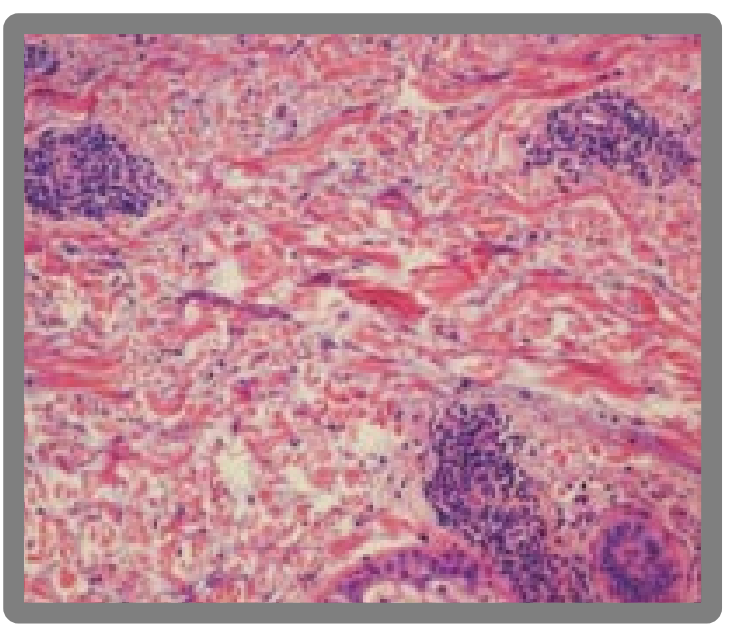

VASCULITIS

\section{REFERENCIAS BIBLIOGRAFICAS}

1. Papiris SA, Moutsopoulos HM: Rare rheumatic disorders: behcet's disease. Baillieres Clin Rheumatol 1993; 7: $173-175$.

2. Yazici H. Behcet's Syndrome. In: Klipple JH, Dieppe PA, eds. Practical Rheumatology. London: Mosby; 1995:393394.

3. Moutsopoulos HM. Behcet's Syndrome. In: Isselbacher K J, Braunwald E, Wilson JD, Martin JB, Fauci AS, Kasper DL, eds. Harrison's Principles of Internal Medi- 
cine, 14E. United States of America, McGraw-Hill Inc; 1998: 2170-2171.

4. Ball EV. Behcet's Syndrome. In: Bennett JC, Plum F, eds. Cecil Textbook of Medicine, 20E. Philadelphia, WB Saunders; 1996:1506-7.

5. Wong RC, Ellis CN, Diaz LA: Behcet's Disease. Int J Dermatol 23: 25, 1984.

6. International Study Group for Behcet's Disease: Criteria for diagnosis of Behcet's Disease. Lancet 1990; 335: 1078 -1080 .

7. Gemignani G, Berrettini S, et al; Hearing and Vestibular Disturbances in Behcet's Syndrome. Ann Otol Rhinol Laryngol 1991; 100:459-463.

8. Soylu L, Aydogan B, Soylu M, Ozsahinoglu C; Hearing Loss in Behcet's

Disease. Ann Otol Rhinol Laryngol 1995; 104: 864-867.

9. Assaad-Khalili S, Sobhy M, Abou-Seif M, El-Sawy M; Cardiac Manifestations

of Behcet's Disease: Clinical, Genetic and Echocardiographic Study;

Elsevier Science Publishers 1993; 481-486.

10.Rokover. Behcet disease: Long-term follow-up of three children and review of literature. Pediatrics 1989; 83: 98692.

11.O'Duffy, JD: Behcet's Disease. In Kelley, WN, Harris, ED, Ruddy, S, and Sledge, CB, eds: Textbook of rheumathology, ed 3, Philadelphia, Wb Saunders Co, 1989. pp 1209 - 1214.

12.Larsson. Treatment of severe colitis in Behcet's syndrome with Thalidomide. J Int Med 1990; 228: 405-407.

13.Chajet, T; and Fainuru, M: Behcet's disease: report of 41 cases and review of the literature, Medicine (Baltimore) 1975; 54: 179-196.

14.Lang. Pediatric onset of Behcet's syndrome with myositis case report and literature review illustrating unusual features. Arthritis and Rheumatism 1990; 33: 418-425.

15.Iragui. Behcet's syndrome presenting as cerebrovascular disease. J Neuro Neurosurg Psychiatry 1986; 49(7): 838-40.

16.Sloper ML, Powell RJ, Dua HS. Tracolimus (FK506) in the Treatment of Posterior Uveitis Refractary to Cyclosporine. Ophtalmology 1999; 106: 723 - 728.

17.Barra C, Belfort R Jr, Abreu MT et al. Behcet Disease in Brazil a review of 49 cases with emphasis on ophtalmic manifestations. Jpn J Ophtalmol 1991; 35:339-46.

18.Giles Cl, and Mazow ML. Uveitis in Children. Am Acad Ophtalmol Otolaryngol 1977. Instruction section, Course 172.
19.Schlaegel TF Jr. Complications of Uveitis. Int Ophtalmol Clin 1977; 17:65-85.

20.Mamo JG. Treatment of Behcet disease with Chlorambucil. A follow-up report. Arch Ophtalmol 1976; 94: 580583.

21. Tricoulis D. Treatment of Behcet's disease with chlorambucil. Br J Ophtalmol 1976; 60: 55-57.

22.Nakae K, Masaki F, Hashimoto T, Inaba G, Mochizuki M, Sakane T. Recent epidemiological features of Behcet's disease in Japan. In: Wechsler B, Godeau P, eds. Behcet's disease. Amsterdam. Excerpta Medica, 1993, 145-51.

23.Zouboulis CC, Kótter I, Djawari D, et al. Epidemiological features of Adamantiades- Behcet's disease in Germany and in Europe. Yonsei Med J 1997; 38: 411-22.

24.Mizuki N, Inoko H, Ohno S, Pathogenic gene responsible for the predisposition to Behcet's disease. Int Rev Immunol 1997; 14: 33-48.

25.Ohno S, Ohguchi M, Hirose S, Matsuda H, Wakisaka A, Aizawa M, Close association of HLA-Bw51 with Behcet's disease. Arch Ophtalmol 1982; 100:1455-8

26.Sakane T. New perspective in Behcet's disease. Int Rev Inmunol 1997; 14: 98-96

27.Zouboulis CC, Büttner P, Djawari D, et al. HLA-Class I antigens in German pacients with AdamantiadesBehcet's disease and correlations with clinical manifestations. In: Wechlser B, Godeau P, eds. Behcet's disease. Amsterdam excerpta Medica, 1993, 175-80.

28.Kastner DL. Intermitent and periodic arthritic syndromes. In: Koopman WJ, ed. Arthritis and allied conditions: a textbook of rheumatology. 13th ed. Vol. 1. Baltimore: Williams \& Wilkins, 1997, 1279-306.

29.Kaklamani VG, Variopoulos G, Kaklamanis PG. Behcet's disease. Semin Arthritis Rheum 1998; 27: 197-217.

30.Ehrlich G. Vasculitis in behcet's disease. Int Rev Inmunol 1997; 107: 241-47.

31.Nussenblatt RB, Palestine AG, Chan CC, Mochizuki and Yancey K, Effectiveness of cyclosporin therapy for behcet's disease. Arthritis Rheum 1985; 28:671-79.

32.Salazar A, Polanco T, Parra B, Duran C. Dificultades diagnósticas en la enfermedad de Behcet. Revista peruana de oftalmología 1989; XV(1): 23-27.

33.Aoki K, Fugioka K, Katsumata H, et Al. Epidemiological studies on Behcet's disease in the Hokkaido district (Japanese). J Clinic Ophthal 1971; 25: 2239.

34.Chajek T, Fainuru J. Behcet's disease: Report of 41 cases and review of the literature. Medicine. 1975; 54: 179.

35.Kalbian VV and Challis MT. Behcet's disease: report of 12 cases with 3 manifesting as papilledema. Am J Med 1970; 49: 823 . 